\title{
Jean De Langeac, Letters and Papers
}

\section{Maurizio Busca}

\section{(2) OpenEdition \\ Journals}

\section{Edizione digitale}

URL: http://journals.openedition.org/studifrancesi/10540

DOI: 10.4000/studifrancesi. 10540

ISSN: 2427-5856

\section{Editore}

Rosenberg \& Sellier

\section{Edizione cartacea}

Data di pubblicazione: 1 décembre 2017

Paginazione: 537-538

ISSN: 0039-2944

\section{Notizia bibliografica digitale}

Maurizio Busca, «Jean De Langeac, Letters and Papers», Studi Francesi [Online], 183 (LXI | |II) | 2017,

online dal 01 février 2018, consultato il 24 janvier 2021. URL: http://journals.openedition.org/

studifrancesi/10540 ; DOI: https://doi.org/10.4000/studifrancesi.10540

\section{Questo documento è stato generato automaticamente il 24 janvier 2021.}

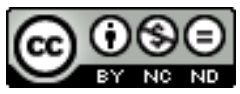

Studi Francesi è distribuita con Licenza Creative Commons Attribuzione - Non commerciale - Non opere derivate 4.0 Internazionale. 


\title{
Jean De Langeac, Letters and Papers
}

\author{
Maurizio Busca
}

\section{NOTIZIA}

JEAN DE LANGEAC, Letters and Papers, edition, commentary and notes by Jan Pendergrass, Genève, Droz, 2016, 643 pp.

1 Vescovo di Avranches e di Limoges, diplomatico incaricato di importanti missioni dagli anni Dieci ai primi anni Quaranta del Xvı secolo, Jean de Langeac è una figura di rilievo del regno di François I alla quale, finora, gli storici si sono interessati soltanto in maniera puntuale. Il lavoro di Pendergrass ha il merito non soltanto di riunire della documentazione inedita o dispersa, ma di fornire un ritratto vivo e sfaccettato di questo personaggio ammirato per le sue doti di ambasciatore, fra gli altri, da Étienne Dolet (cui fu legato da rapporti professionali). Il cuore del volume è rappresentato dall'edizione commentata di una scelta di 150 lettere e documenti amministrativi di varia natura, inviati o ricevuti da Langeac, redatti o tradotti in lingua francese, latina, italiana, tedesca o portoghese: istruzioni e rapporti di missione, discorsi, dichiarazioni di spesa, lettere di nomina per vari incarichi, ricevute, atti di possesso di proprietà e di investiture, registrazioni di controversie, certificati di tonsura, i verbali di una riunione del Grand Conseil di François I, un certificato di traslazione di reliquie, un contratto per servizi artistici e vari moduli amministrativi pre-stampati emessi dagli esattori del vescovo a Limoges. Ogni documento è presentato da un'introduzione ed è accompagnato da un apparato critico e da cospicue note storiche e linguistiche. Nella loro estrema varietà, i materiali raccolti permettono di ricostruire le responsabilità di Langeac nella gestione dei suoi numerosi incarichi, la sua rete di legami personali e professionali, ma anche la natura della sua formazione.

2 La breve ma densa introduzione del curatore (pp.19-37) ripercorre le vicende biografiche di Langeac alla luce dei documenti editi, proponendo al contempo delle osservazioni sullo stile epistolare e sulle pratiche di scrittura del vescovo e dei suoi collaboratori. Questa prima sezione è seguita da un'utile cronologia ragionata 
(pp. 39-49) nella quale ad ogni data sono associati riferimenti bibliografici e rimandi ai testi inseriti nel volume. In una seconda tabella cronologica (pp. 51-53) sono elencate le missioni compiute da Langeac per conto della Chiesa e dello Stato fra il 1511 e il 1541 in Francia, Italia, Spagna, Portogallo, Svizzera, Polonia e Scozia, per ognuna delle quali sono segnalate le principali tappe e le distanze percorse. Una nutrita bibliografia (pp. 575616) e un indice nei nomi di persona e di luogo (pp. 617643) completano il volume.

3 Segnaliamo che al presente lavoro farà seguito una raccolta di Letters and Papers (1527-1555) del parlamentare e diplomatico Claude Dodieu, sempre a cura di Jan Pendergrass, attualmente in lavorazione. 